\title{
A new quasi-exactly solvable problem and its connection with an anharmonic oscillator
}

\author{
Da-Bao Yang, Fu-Lin Zhang, and Jing-Ling Chen* \\ Chern Institute of Mathematics, Nankai University, Tianjin 300071, China
}

(Dated: May 31, 2022)

\begin{abstract}
The two-dimensional hydrogen with a linear potential in a magnetic field is solved by two different methods. Furthermore the connection between the model and an anharmonic oscillator is investigated by methods of KS transformation.

PACS numbers:
\end{abstract}

*Electronic address: chenj1@nankai.edu.cn 


\section{INTRODUCTION}

The discovery of quasi-exactly solvable quantal problems was a remarkable contribution in last century[1]. This kind of problem could be solved by lie algebraic methods, which was pioneered by Turbiner[2]. The method was also be applied to an anharmonic oscillators by Chen et. al.[3]. Moreover a generalization of the basic lie algebraic construction was considered by Shifman[4]. You can also confer to a reviewed paper by Shifman[1]. However, the quasi-exactly solvable problem could also be calculated by an analytical way[5].

This paper is concentrated on a model that is a two-dimensional hydrogen with a linear potential in a homogeneous magnetic field. This model is very meaningful among the quasiexactly problems. First, its potential is novel and complex, moreover when the coefficient of the linear potential becomes zero, its all result could go back to the simpler circumstance which have been investigated by Taut[5]. Second, the sl(2) algebra structure hidden in the Hamiltonian is not apparent, however it will be revealed in this paper. Third, this model is also solved by an analytical method whose result coincide with the one by the sl(2) method. Last but not least, a new sextic oscillator with a centrifugal potential is obtained from this model. Furthermore, the connection between them is constructed.

This paper is organised as follows: in section two, the separation of variables in the corresponding schrödinger equation is displayed, so we get the radial part equation; in section three, we solve it by the lie-algebraic methods, i.e., $\operatorname{sl}(2)$; in section four, it is solved by an analytic way, therefore, the two results coincide with each other; in section five we build its connection with the sextic oscillator with a centrifugal barrier; in section six, a conclusion is drawed.

\section{THE SEPARATION OF VARIABLES}

Now, we consider a two-dimensional hydrogen with a linear potential in a homogeneous magnetic field, where the magnetic field $\vec{B}$ is perpendicular to the plan in which the electron is located. The schrödinger equation reads

$$
\left[\frac{1}{2}\left(\vec{p}+\frac{1}{c} \overrightarrow{\mathcal{A}}\right)^{2}-\frac{Z}{r}+k r\right] \psi=E \psi
$$

where $c$ is the velocity of light and the vector potential in the symmetric gauge is $\overrightarrow{\mathcal{A}}=\frac{1}{2} \vec{B} \times \vec{r}$. Under some operation, the above equation can be transformed to be 


$$
\frac{1}{2}\left[\vec{p}^{2} \psi+\frac{1}{c}(\vec{p} \cdot \overrightarrow{\mathcal{A}}) \psi+\frac{2}{c} \overrightarrow{\mathcal{A}} \cdot \vec{p} \psi+\frac{1}{c^{2}} \overrightarrow{\mathcal{A}}^{2} \psi\right]-\frac{Z}{r} \psi+k r \psi=E \psi
$$

In polar coordinates, letting $r$ and $\theta$ represent the radius and azimuthal angle respectively, we can get

$$
\left\{\begin{array}{l}
\vec{p}=-i\left(\overrightarrow{e_{r}} \frac{\partial}{\partial r}+\overrightarrow{e_{\theta}} \frac{1}{r} \frac{\partial}{\partial \theta}\right) \\
\overrightarrow{\mathcal{A}}=\frac{1}{2} \vec{B} \times \vec{r}=\frac{1}{2} B r \overrightarrow{e_{\theta}} \\
\vec{p} \cdot \overrightarrow{\mathcal{A}}=0 \\
\overrightarrow{\mathcal{A}} \cdot \vec{p}=-i \frac{1}{2} B \frac{\partial}{\partial \theta} \\
\overrightarrow{\mathcal{A}^{2}}=\frac{1}{4} B^{2} r^{2}
\end{array}\right.
$$

Substitution the above equations into the Eq.(1), we get

$$
\frac{1}{2}\left[-\nabla^{2} \psi-i 2 \omega_{L} \frac{\partial}{\partial \theta} \psi+\omega_{L}^{2} r^{2} \psi\right]-\frac{Z}{r} \psi+k r \psi=E \psi
$$

where $\nabla^{2}=\frac{1}{r} \frac{\partial}{\partial r} r \frac{\partial}{\partial r}+\frac{1}{r^{2}} \frac{\partial}{\partial \theta^{2}}$ and $\omega_{L}=\frac{B}{2 c}$. Having represented the equation in a concrete coordinate, we will separate the variables in the next paragraph.

Substituting

$$
\psi(r, \theta)=\frac{1}{\sqrt{2 \pi}} e^{i m \theta} R(r)
$$

into the above equation, where $m=0, \pm 1, \pm 2, \ldots$, we get the radial part equation, which is

$$
\left(-\frac{1}{2} \frac{d^{2}}{d r^{2}}-\frac{1}{2} \frac{1}{r} \frac{d}{d r}+\frac{\omega_{L}^{2} r^{2}}{2}+k r+\omega_{L} m-\frac{Z}{r}+\frac{m^{2}}{2 r^{2}}\right) R=E R .
$$

The above differential equation will be solved by the method of $\operatorname{sl}(2)$ algebra in the next secion.

\section{SOLVING THE RADIAL EQUATION WITH THE SL(2) ALGEBRA}

First of all, let us recall the essential idea that the schrödinger equation is solved by $\operatorname{sl}(2)$. The schrödinger equation determining the stationary states of the system is

$$
\begin{gathered}
H \psi(r)=E \psi(r), \\
H=-\frac{1}{2} \frac{d^{2}}{d r^{2}}+V(r) .
\end{gathered}
$$

According to [1], we can perform a quasi-gauge transformation 


$$
\psi(r)=\tilde{\psi}(r) \exp \left(-\int A(r) d r\right)
$$

Then the stationary schrödinger equation can be transformed to

$$
\begin{gathered}
H{ }^{\prime \prime}, \tilde{\psi}=E \tilde{\psi}, \\
H_{" G "}=-\frac{1}{2}\left(\frac{d}{d x}-A(x)\right)^{2}+V(x) .
\end{gathered}
$$

Furthermore $H$ "G" can be represented by the combination of the partial algebraization of the generators of $\operatorname{sl}(2)$, which is

$$
H " G "=\sum_{a, b= \pm, 0} C_{a b} T^{a} T^{b}+\sum_{a= \pm, 0} C_{a} T^{a}+\text { const }
$$

where

$$
\begin{gathered}
T^{+}=2 j r-r^{2} \frac{d}{d r} \\
T^{0}=-j+r \frac{d}{d r} \\
T^{-}=\frac{d}{d r} .
\end{gathered}
$$

It is very easy to check that the commutation relations for $T^{ \pm}$and $T^{0}$ are indeed those inherent to the sl(2) algebra:

$$
\left[T^{+}, T^{-}\right]=2 T^{0}, \quad\left[T^{0}, T^{+}\right]=+T^{+}, \quad\left[T^{0}, T^{-}\right]=-T^{-} .
$$

Finally, with the aid of the above commutation relations, the transformed Hamiltonian $H^{\prime}$ " can be represented by a $(2 j+1) \times(2 j+1)$ matrix, whose basis are

$$
\{\tilde{\psi}\}=\left\{1, r, r^{2}, \cdots, r^{2 j-1}, r^{2 j}\right\}
$$

Then the standard algorithm of diagonalization of the finite matrix gives us $(2 j+1)$ eigenvalues and the corresponding eigenfunctions of the transformed Hamiltonian.

Blindly following the program outlined above, we do not find the above model that we pay attention to in the section 2 can be solved by the methods of $\mathrm{sl}(2)$, in another word, the Hamiltonian $H$ isn't reducible to the quadratic combination of the $\operatorname{sl}(2)$ generators. However, we can consider a corresponding eigenvalue problem, $f(r) H \psi=E \psi$, where $f$ 
is an appropriately chosen function of the variable $r$, so that $f H$ can be reducible to the standard form (6). The above explanation maybe a litter obscure, moreover this method will be showed in more transparent terms in the following paragraphs.

Let us introduce an auxiliary Hamiltonian

$$
\tilde{H}=\gamma\left(-\frac{1}{2} \frac{d^{2}}{d r^{2}}-\frac{1}{2 r} \frac{d}{d r}+\frac{\omega_{L}^{2} r^{2}}{2}+\omega_{L} m+\frac{m^{2}}{2 r^{2}}\right)-\frac{Z}{r}+\gamma k r-\gamma X
$$

where $\gamma$ and $X$ are constant coefficients.Next, we calculate the "gauge"-transformed Hamiltonian $\tilde{H}^{\prime}$, ", simply stemming form $\tilde{H}$ after the substitution

$$
\frac{d}{d r} \rightarrow \frac{d}{d r}-A
$$

Then

$\tilde{H}_{" G},=\gamma\left(-\frac{1}{2} \frac{d^{2}}{d r^{2}}-\frac{1}{2 r} \frac{d}{d r}+A \frac{d}{d r}\right)-\frac{1}{2} \gamma A^{2}+\gamma \frac{\omega_{L}^{2} r^{2}}{2}+\gamma k r+\frac{1}{2} \gamma A^{\prime}+\gamma \frac{A}{2 r}+\gamma \frac{m^{2}}{2 r^{2}}-\frac{Z}{r}+\gamma\left(m \omega_{L}-X\right)$.

After a short analysis, we can get a conclusion that: when $r \rightarrow \infty$, the term $\gamma \frac{\omega_{L}^{2} r^{2}}{2}+\gamma k r$ will become the dominant one; when $r \rightarrow 0$, the term $\gamma \frac{m^{2}}{2 r^{2}}$ will become the dominant counterpart. In order to guarantee the normalizability of the wave function, we must choose a proper form of $A$ to cancel out the above terms. A short reflection shows that

$$
A=\mu r+\delta+\frac{\nu}{r}
$$

Substituting the above Eq.(11) into Eq.(10), one gets

$$
\begin{aligned}
\tilde{H} " G^{\prime \prime}= & \gamma\left(-\frac{1}{2} \frac{d^{2}}{d r^{2}}-\frac{1}{2 r} \frac{d}{d r}+A \frac{d}{d r}\right)+\frac{1}{2} \gamma\left(\omega_{L}^{2}-\mu^{2}\right) r^{2}+\gamma(k-\mu \delta) r+\left(\frac{1}{2} \gamma \delta-\gamma \delta \nu-Z\right) \frac{1}{r} \\
& +\frac{1}{2} \gamma\left(m^{2}-\nu^{2}\right) \frac{1}{r^{2}}+\gamma\left(+\omega_{L} m+\mu-\mu \nu-\frac{1}{2} \delta^{2}-X\right)
\end{aligned}
$$

Moreover, from above analysis, one knows the coefficients of $r^{2}, r$ and $\frac{1}{r^{2}}$ are equal to 0 respectively, which are

$$
\left\{\begin{array}{l}
\omega_{L}^{2}-\mu^{2}=0 \\
k-\mu \delta=0 \\
m^{2}-\nu^{2}=0
\end{array}\right.
$$

The above equations can be solved out:

$$
\left\{\begin{array}{l}
\mu= \pm \omega_{L} \\
\delta=\frac{k}{\mu} \\
\nu= \pm|m|
\end{array}\right.
$$


Substituting Eq.(11) and Eq.(13) into Eq.(14), one finds that only

$$
R(r)=R \tilde{R}(r) \exp \left(-\frac{1}{2} \omega_{L} r^{2}-\frac{k}{\omega_{L}} r+|m| \ln r\right)
$$

can guarantee that the wave function converge at both sides of $r \rightarrow 0$ and $r \rightarrow \infty$ (Note that we replace $\psi(r)$ and $\tilde{\psi}(r)$ by $R(r)$ and $\tilde{R}(r)$ respectively. $)$, that is to say

$$
\left\{\begin{array}{l}
\mu=+\omega_{L} \\
\delta=\frac{k}{\omega_{L}} \\
\nu=-|m|
\end{array}\right.
$$

Substituting Eq.(15) into Eq.(12), one can gets

$$
\frac{r}{\gamma} \tilde{H}_{" G}, \tilde{R}=\frac{E(\gamma)}{\gamma} \tilde{R}
$$

where

$$
\begin{gathered}
\tilde{H} " G "=\gamma\left[-\frac{1}{2} \frac{d^{2}}{d r^{2}}-\frac{1}{2 r} \frac{d}{d r}+\left(\omega_{L} r+\frac{k}{\omega_{L}}-\frac{|m|}{r}\right) \frac{d}{d r}\right] \\
+\left[\gamma \frac{k}{\omega_{L}}\left(|m|+\frac{1}{2}\right)-Z\right]+\gamma\left[\omega_{L}(1+m+|m|)-\frac{1}{2}\left(\frac{k}{\omega_{L}}\right)^{2}-X\right] .
\end{gathered}
$$

The reason that I divided both sides of Eq.(16) by $\gamma$ is for a convenient calculation. The reader maybe puzzled by the reason why we use $r$ to multiple $\tilde{H}$ " $G$ ", because $r \tilde{H}$ " "G" can reducible to a quadratic combination of the generator of $\mathrm{sl}(2)$.Under the inverse transformation Eq.(9), the Eq.(16) can be transformed to be

$$
\frac{1}{\gamma} \tilde{H} R(r)=\frac{E(\gamma)}{\gamma r} R(r)
$$

where

$$
R=\tilde{R} \exp \left(-\frac{1}{2} \omega_{L} r^{2}-\frac{k}{\omega_{L}} r+|m| \ln r\right)
$$

Substituting Eq.(8) into Eq.(17), we can get

$$
\left(-\frac{1}{2} \frac{d^{2}}{d r^{2}}-\frac{1}{2 r} \frac{d}{d r}+\frac{\omega_{L}^{2} r^{2}}{2}+k r+\omega_{L} m-\frac{1}{\gamma} \frac{Z+E}{r}+\frac{m^{2}}{2 r^{2}}\right) R=X R .
$$

By comparison with the Eq.(4), we observe that we have actually solved the original problem provided that the parameter $\gamma$ is chosen as follows

$$
\frac{Z+E}{\gamma}=Z
$$

And we represented the eigenvalue by another symbol $X$ instead. 
In order to solve Eq.(18) by sl(2), let's return to Eq.(16). Following what I said before, the $\frac{r}{\gamma} \tilde{H}^{\prime} G$ " can be reducible to the combination of generators of $\operatorname{sl}(2)$, which is

$$
\frac{r}{\gamma} \tilde{H} " G "=C_{1} T^{0} T^{-}+C_{2} T^{+}+C_{3} T^{0}+C_{4} T^{-}+C_{0} .
$$

Substituting Eq.(7) into the above Eq.(20), we can get

$$
\frac{r}{\gamma} \tilde{H}^{\prime} G^{\prime \prime}=C_{1} r \frac{d^{2}}{d r^{2}}-C_{2} r^{2} \frac{d}{d r}+C_{3} r \frac{d}{d r}+\left(C_{4}-j C_{1}\right) \frac{d}{d r}+2 C_{2} j r-j C_{3+} C_{0} .
$$

By comparison with Eq.(16), the coefficients can be solved from equations

$$
\left\{\begin{array}{l}
C_{1}=-\frac{1}{2} \\
-C_{2}=\omega_{L} \\
C_{3}=\frac{k}{\omega_{L}} \\
C_{4}-j C_{1}=-|m|-\frac{1}{2} \\
2 C_{2} j=\omega_{L}(1+m+|m|)-X \\
-j C_{2}+C_{0}=\frac{k}{\omega_{L}}\left(\frac{1}{2}+|m|\right)-\frac{Z}{r}
\end{array}\right.
$$

A further simplified calculation show that

$$
\left\{\begin{array}{l}
C_{1}=-\frac{1}{2} \\
C_{2}=-\omega_{L} \\
C_{3}=\frac{k}{\omega_{L}} \\
C_{4}=-\frac{1}{2}(1+j+2|m|) \\
X=\omega_{L}(2 j+1+m+|m|)-\frac{1}{2}\left(\frac{k}{\omega_{L}}\right)^{2} \\
C_{0}=\frac{k}{\omega_{L}}\left(|m|+\frac{1}{2}+j\right)-\frac{z}{\gamma}
\end{array}\right.
$$

Substituting the above Eq.(21) and Eq.(20) into Eq.(16), one gets

$$
\left[-\frac{1}{2} T^{0} T^{-}-\omega_{L} T^{+}+\frac{k}{\omega_{L}} T^{0}-\frac{1}{2}(1+j+|m|) T^{-}+\frac{k}{\omega_{L}}\left(|m|+\frac{1}{2}+j\right)\right] \tilde{R}=\frac{Z+E}{\gamma} \tilde{R}
$$

By use of the constraint which is Eq.(19), the above Eq.(22) can be transformed to

$$
\left[-\frac{1}{2} T^{0} T^{-}-\omega_{L} T^{+}+\frac{k}{\omega_{L}} T^{0}-\frac{1}{2}(1+j+|m|) T^{-}+\frac{k}{\omega_{L}}\left(|m|+\frac{1}{2}+j\right)\right] \tilde{R}=Z \tilde{R}
$$

1) $j=0$ 
Hence, we know

$$
\tilde{R}=a_{0}
$$

Substituting Eq.(24) into Eq.(14), one get

$$
R=a_{0} \exp \left(-\frac{1}{2} \omega_{L} r^{2}-\frac{k}{\omega_{L}} r+|m| \ln r\right) .
$$

Substituting Eq.(7) and Eq.(24) into Eq.(23), we can get

$$
\frac{k}{\omega_{L}}\left(|m|+\frac{1}{2}\right)=Z,
$$

which is precisely the constraint of eigenvalues. Substituting the above Eq.(26) into the fifth Equation of Eq.(21), we can get the eigenvalue

$$
X_{0}=\omega_{L}(1+m+|m|)-\frac{1}{2}\left(\frac{Z}{|m|+\frac{1}{2}}\right)^{2}
$$

2) $j=\frac{1}{2}$

It is very easy to calculate that

$$
T^{+}=\left(\begin{array}{ll}
0 & 1 \\
0 & 0
\end{array}\right) \quad T^{0}=\frac{1}{2}\left(\begin{array}{cc}
1 & 0 \\
0 & -1
\end{array}\right) \quad T^{-}=\left(\begin{array}{ll}
0 & 0 \\
1 & 0
\end{array}\right) .
$$

Substitution the above Eq.(27) into Eq.(23), we can get

$$
\left(\begin{array}{cc}
\frac{k}{\omega_{L}}\left(|m|+\frac{3}{2}\right) & -\omega_{L} \\
-\left(|m|+\frac{1}{2}\right) & \frac{k}{\omega_{L}}\left(|m|+\frac{1}{2}\right)
\end{array}\right)\left(\begin{array}{l}
a_{1} \\
a_{0}
\end{array}\right)=Z\left(\begin{array}{l}
a_{1} \\
a_{0}
\end{array}\right) .
$$

Such a equation has a nontrivial solution if the determinant of the corresponding matrix vanishes, i.e.

$$
\left|\begin{array}{cc}
\frac{k}{\omega_{L}}\left(|m|+\frac{3}{2}\right)-Z & -\omega_{L} \\
-\left(|m|+\frac{1}{2}\right) & \frac{k}{\omega_{L}}\left(|m|+\frac{1}{2}\right)-Z
\end{array}\right|=0 .
$$

The equation can be further simplified to be

$$
\left.\omega_{L}^{2} Z^{2}-2 k \omega_{L}(|m|+1) Z+k^{2}\left(|m|+\frac{3}{2}\right)\left(|m|+\frac{1}{2}\right)-\omega_{L}^{3}\left(|m|+\frac{1}{2}\right)\right]=0 .
$$

Form it, we can draw a conclusion that the parameters $\omega_{L}$ and $k$ can't be chosen arbitrarily, they must satisfy the above Eq.(29). The above Eq.(29) can be regarded as a quadratic Equation about $Z$. As a result, the solution of it can be expressed as

$$
Z=\frac{k(|m|+1) \pm \sqrt{M}}{\omega_{L}}
$$


where $M \equiv \frac{k^{2}}{4}+\omega_{L}^{3}\left(\frac{1}{2}+|m|\right)$. In the following paragraph, we will calculate the different eigenfunctions corresponding to different eigenvalues.

a) $Z=\frac{k(|m|+1)+\sqrt{M}}{\omega_{L}}$,

Substituting the above equation into Eq.(28), we get

$$
a_{0}\left(\begin{array}{c}
-\frac{\frac{1}{2} \frac{k}{\omega_{L}}+\frac{1}{\omega_{L}} \sqrt{M}}{\frac{1}{2}+|m|} \\
1
\end{array}\right) \equiv a_{0}\left(1-\frac{\frac{1}{2} \frac{k}{\omega_{L}}+\frac{1}{\omega_{L}} \sqrt{M}}{\frac{1}{2}+|m|} r\right) .
$$

Substituting the above Eq.(31) into Eq.(14)

$$
R(r)_{j=\frac{1}{2}}^{m_{j}=\frac{1}{2}}=a_{0}\left(1-\frac{\frac{1}{2} \frac{k}{\omega_{L}}+\frac{1}{\omega_{L}} \sqrt{M}}{\frac{1}{2}+|m|} r\right) \exp \left(-\frac{1}{2} \omega_{L} r^{2}-\frac{k}{\omega_{L}} r+|m| \ln r\right) .
$$

b) $Z=\frac{k(|m|+1)-\sqrt{M}}{\omega_{L}}$,

Following the above procedure, we get the radial part of the wave function, i.e.,

$$
R(r)_{j=\frac{1}{2}}^{m_{j}=-\frac{1}{2}}=a_{0}\left(1-\frac{\frac{1}{2} \frac{k}{\omega_{L}}-\frac{1}{\omega_{L}} \sqrt{M}}{\frac{1}{2}+|m|} r\right) \exp \left(-\frac{1}{2} \omega_{L} r^{2}-\frac{k}{\omega_{L}} r+|m| \ln r\right) .
$$

With the constraint of Eq.(29), the eigenvalue of energy becomes

$$
X_{\frac{1}{2}}=\omega_{L}(2+m+|m|)-\frac{1}{2}\left(\frac{k}{\omega_{L}}\right)^{2}
$$

3)Arbitrary j

According to the choice of the phase, we know the following formulation, i.e.,

$$
T^{ \pm}|j m\rangle=\sqrt{(j \mp m)(j \pm m+1)}|j, m \pm 1\rangle \quad T^{0}|j m\rangle=m|j m\rangle .
$$

The $j_{ \pm}$and $j_{0}$ can be represented in the following $(2 j+1) \times(2 j+1)$ matrix,

$$
T^{+}=\left(\begin{array}{cccccc}
0 & \sqrt{1 \cdot 2 j} & 0 & & 0 & 0 \\
0 & 0 & \sqrt{2 \cdot(2 j-1)} & 0 & 0 \\
\vdots & \vdots & \vdots & \ddots & \vdots & \vdots \\
0 & 0 & 0 & \cdots & \sqrt{(2 j-1) \cdot 2} & 0 \\
0 & 0 & 0 & & 0 & \sqrt{2 j \cdot 1} \\
0 & 0 & 0 & & 0 & 0
\end{array}\right)
$$




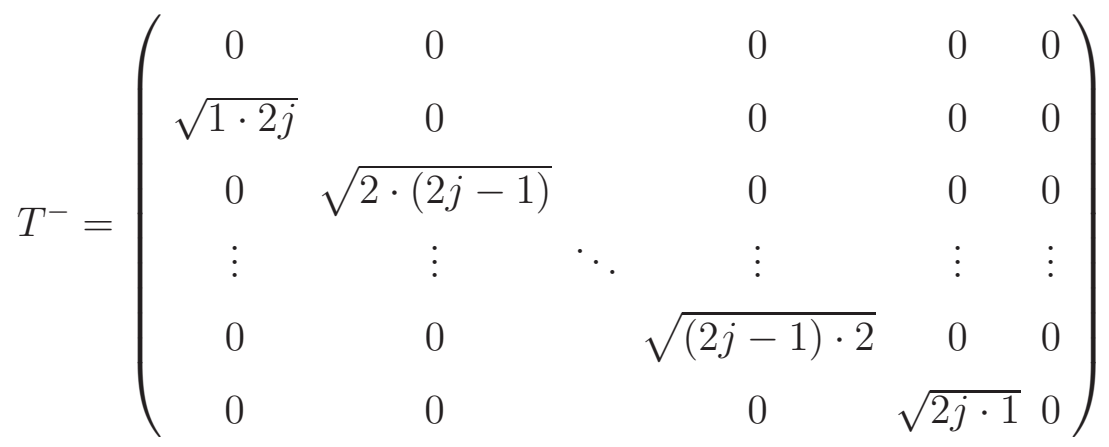

$$
\begin{aligned}
& T^{0}=\left(\begin{array}{lllll}
j & & & & \\
\\
& j-1 & & & \\
& & j-2 & & \\
& & \ddots & & \\
& & & -j+1 & \\
& & & & -j
\end{array}\right)
\end{aligned}
$$

Substitution the above Eq.(34), Eq.(35) and Eq.(36) into Eq.(23), so the problem is deduced to an eigenvalue and eigenfunction problem. In order to get the nontrivial eigenfunction, the determinant of the corresponding matrix must vanish, which will give a onstraint about $\omega_{L}$ and $k$.Similarly, we will get the radial part of the wave function. Furthermore, the fifth equation of Eq.(21) tell us the eigenvalue of energy,

$$
X_{j}=\omega_{L}(2 j+1+m+|m|)-\frac{1}{2}\left(\frac{k}{\omega_{L}}\right)^{2} .
$$

To sum up, in this section, we have solved Eq.(4) by method of $\mathrm{sl}(2)$. Moreover, we will solved it again by another method, namely, an analytical methods.

\section{SOLVING THE SAME EQUATION WITH ANALYTICAL METHOD}

In order to solve Eq.(4), using the following transformation, i.e.

$$
R=\frac{u}{\sqrt{r}}
$$

we can get

$$
-\frac{1}{2} \frac{d^{2} u}{d r^{2}}+\frac{1}{2}\left(m^{2}-\frac{1}{4}\right) \frac{1}{r^{2}} u-\frac{Z}{r} u+\omega_{L} m u+k r u+\frac{1}{2} \omega_{L}^{2} r^{2} u=E u .
$$

Due to the condition of square-integrability of the wave function, it is useful to consider of the limits $r \rightarrow 0$ and $r \rightarrow \infty$ of the above Eq.(38). For $r \rightarrow 0$, the term containing $\frac{1}{r^{2}}$ is 
dominant and we get the equation

$$
-\frac{1}{2} \frac{d^{2} u}{d r^{2}}+\frac{1}{2}\left(m^{2}-\frac{1}{4}\right) \frac{1}{r^{2}} u=0 .
$$

Because the above Eq.(39) contains a normal singularity, so we substitute a power series $u=r^{s} \sum_{i=0}^{\infty} b_{i} r^{i}$ into the equation and one gets

$$
-\sum_{i=0}^{\infty}(s+i)(s+i-1) b_{i} r^{s+i-2}+\left(m^{2}-\frac{1}{4}\right) \sum_{i=0}^{\infty} b_{i} r^{s+i-2}=0 .
$$

Due to the uniqueness of the series, the coefficient of it is equal to zero, we can get

$$
-(s+i)(s+i-1) b_{i}+\left(m^{2}-\frac{1}{4}\right) b_{i}=0 .
$$

By use of the limits $r \rightarrow 0$, only the lowest-order term become dominant, so we omits the higher order terms and get

$$
-s(s-1) b_{0}+\left(m^{2}-\frac{1}{4}\right) b_{0}=0 .
$$

In order to get the nontrivial solution, we must set $b_{0} \neq 0$, so one gets $s=m+\frac{1}{2}$ or $s=-m+\frac{1}{2}$. However, if $\mathrm{m}$ become negative, the solution diverges when $r \rightarrow 0$. Hence, we get

$$
s=|m|+\frac{1}{2}
$$

So the solution of Eq.(39) is

$$
u=r^{|m|+\frac{1}{2}} .
$$

Having solved the solution $r \rightarrow 0$, we consider about another situation $r \rightarrow \infty$. Under this circumstance, the terms containing $r^{2}$ and $r$ in Eq.(38) become dominant. So the equation becomes

$$
-\frac{1}{2} \frac{d^{2} u}{d r^{2}}+\frac{1}{2} \omega_{L}^{2} r^{2} u+k r u=0 .
$$

We can verify the solution of the above equation is

$$
u=\exp \left(-\frac{1}{2} \omega_{L} r^{2}-\frac{k}{\omega_{L}} r\right)
$$

Considering both Eq.(40) and Eq.(41), we can write the solution of Eq.(38)as

$$
u=r^{|m|+\frac{1}{2}} e^{-\frac{1}{2} \omega_{L} r^{2}-\frac{k}{\omega_{L}} r} \varphi .
$$

Substituting the above Eq.(42)into (38), we get

$$
\begin{gathered}
-\frac{1}{2} r \frac{d^{2} \varphi}{d r^{2}}+\omega_{L} r^{2} \frac{d \varphi}{d r}+\frac{k}{\omega_{L}} r \frac{d \varphi}{d r}-\left(|m|+\frac{1}{2}\right) \frac{d \varphi}{d r}+\left(|m| \omega_{L}+\omega_{L}+m \omega_{L}-\frac{1}{2} \frac{k^{2}}{\omega_{L}^{2}}-E\right) r \varphi \\
+\left[\left(|m|+\frac{1}{2}\right) \frac{k}{\omega_{L}}-Z\right] \varphi=0 .
\end{gathered}
$$


The above differential equation has two nonessential singularities at $r=0, \infty$. It's index equation takes this form

$$
s(s+2|m|)=0 .
$$

It's solutions are 0 and $-2|m|$ respectively. However, if $s=-2|m|$, the solution diverges, in another words, it violate the demand of square-integrability of the wave function. Hence, $s$ must be 0 . Therefore, we can substitute the power series

$$
\varphi=\sum_{n=0}^{\infty} a_{n} r^{n}
$$

into Eq.(43)and find the recurrence relation

$$
\begin{gathered}
-\frac{1}{2} \sum_{n=1}^{\infty} a_{n+1}(n+1) n r^{n}+\omega_{L} \sum_{n=2}^{\infty} a_{n-1}(n-1) r^{n}+\frac{k}{\omega_{L}} \sum_{n=1}^{\infty} a_{n} n r^{n} \\
-\left(|m|+\frac{1}{2}\right) \sum_{n=0}^{\infty} a_{n+1}(n+1) r^{n}+\left(|m| \omega_{L}+\omega_{L}+m \omega_{L}-\frac{1}{2} \frac{k^{2}}{\omega_{L}^{2}}-E\right) \sum_{n=1}^{\infty} a_{n-1} r^{n} \\
+\left[\left(|m|+\frac{1}{2}\right) \frac{k}{\omega_{L}}-Z\right] \sum_{n=0}^{\infty} a_{n} r^{n}=0
\end{gathered}
$$

Let us discuss in detail in the following paragraphs.

$$
\text { 1) } n=0
$$

$$
a_{1}=\frac{\left(|m|+\frac{1}{2}\right) \frac{k}{\omega_{L}}-Z}{|m|+\frac{1}{2}} a_{0} .
$$

We put forward a hypothesis that the ground state exists, so we get $a_{0} \neq 0, a_{1}=0, a_{2}=0$, .... However, conferring to Eq.(46), if $a_{0} \neq 0, a_{2}$ may be not equal to 0 , which is contradict the hypothesis. So we can draw a conclusion that $a_{0}=0$, that is to say, there isn't a ground state.

$$
\text { 2) } n=1
$$

$$
a_{2}=\frac{|m| \omega_{L}+\omega_{L}+m \omega_{L}-\frac{1}{2} \frac{k^{2}}{\omega_{L}^{2}}-E}{2(|m|+1)} a_{0}+\frac{\left(|m|+\frac{3}{2}\right) \frac{k}{\omega_{L}}-Z}{2(|m|+1)} a_{1} .
$$

If the first excited state exists, $a_{0}$ and $a_{1}$ can't be equal to 0 simultaneously, $a_{2}=0, a_{3}=0$, … Conferring to Eq.(49), $a_{1}$ will have a effect on $a_{3}$, so $a_{1}=0$, that is to say, conferring to Eq. $(45)$,

$$
Z=\left(|m|+\frac{1}{2}\right) \frac{k}{\omega_{L}}
$$

So the radial part of wave function is

$$
R_{n=1}=r^{|m|} e^{-\frac{1}{2} \omega_{L} r^{2}-\frac{k}{\omega_{L}} r} a_{0},
$$


which is gained by use of Eq.(37) and Eq.(42).By comparison with Eq.(25), it is found easily that the two results coincide with each other. From above analysis, we can draw a conclusion that $a_{0} \neq 0, a_{1}=0, a_{2}=0, \cdots$, conferring to Eq.(46), so we get

$$
E_{1}=\omega_{L}(1+|m|+m)-\frac{1}{2} \frac{k^{2}}{\omega_{L}^{2}}
$$

3) $n=2$

$$
a_{3}=\frac{|m| \omega_{L}+2 \omega_{L}+m \omega_{L}-\frac{1}{2} \frac{k^{2}}{\omega_{L}^{2}}-E}{3\left(|m|+\frac{3}{2}\right)} a_{1}+\frac{\left(|m|+\frac{5}{2}\right) \frac{k}{\omega_{L}}-Z}{3\left(|m|+\frac{3}{2}\right)} a_{2} .
$$

Following the above analysis, if the second excited state exists, we can get $a_{3}=0, a_{4}=0$ and so on. It is necessary to demand the first term and the second term are equal to 0 respectively, conferring to Eq.(49). Moreover if $a_{2} \neq 0$, it may render $a_{4} \neq 0$, which is contradict our hypothesis. So we must let $a_{2}=0$, in another word, the second term become zero already. Now let us concentrate on the first term. If $a_{1}=0$, this case will be the same with the first excited state. So we can get

$$
E_{2}=(2+|m|+m) \omega_{L}-\frac{1}{2} \frac{k^{2}}{\omega_{L}^{2}} .
$$

Substituting Eq.(45) and Eq.(50) into Eq.(46), we can get

$$
a_{2}=\frac{\left[\left(|m|+\frac{3}{2}\right) \frac{k}{\omega_{L}}-Z\right]\left[\left(|m|+\frac{1}{2}\right) \frac{k}{\omega_{L}}-Z\right]-\left(|m|+\frac{1}{2}\right) \omega_{L}}{2(|m|+1)\left(|m|+\frac{1}{2}\right)} a_{0} .
$$

From above analysis, we know $a_{2}=0$, that is to say,

$$
\left[\left(|m|+\frac{3}{2}\right) \frac{k}{\omega_{L}}-Z\right]\left[\left(|m|+\frac{1}{2}\right) \frac{k}{\omega_{L}}-Z\right]-\left(|m|+\frac{1}{2}\right) \omega_{L}=0,
$$

which coincide with Eq.(29). Therefore, regarding $Z$ as unknown root, we can certainly get the same solution with Eq.(29), i.e., Eq.(30). Substituting Eq.(42) and Eq.(44) into Eq.(37), we can also get the radial part of the wave function,

$$
R_{n=2}=r^{|m|} e^{-\frac{1}{2} \omega_{L} r^{2}-\frac{k}{\omega_{L}} r}\left[\frac{\left(|m|+\frac{1}{2}\right) \frac{k}{\omega_{L}}-Z}{|m|+\frac{1}{2}} r+1\right] a_{0} .
$$

Substituting Eq.(30) into the above Eq.(51), we get

$$
R_{n=2}^{ \pm}=r^{|m|} e^{-\frac{1}{2} \omega_{L} r^{2}-\frac{k}{\omega_{L}} r}\left[1-\frac{\frac{1}{2} \frac{k}{\omega_{L}} \pm \frac{1}{\omega_{L}} \sqrt{M}}{|m|+\frac{1}{2}} r\right] a_{0}
$$

which is coincide with Eq.(32) and Eq.(33). 
4) $n \geq 2$

$$
a_{n+1}=\frac{\omega_{L}(n+|m|+m)-\frac{1}{2} \frac{k^{2}}{\omega_{L}^{2}}-E}{(n+1)\left(|m|+\frac{1+n}{2}\right)} a_{n-1}+\frac{\left(|m|+\frac{1}{2}+n\right) \frac{k}{\omega_{L}}-Z}{(n+1)\left(|m|+\frac{1+n}{2}\right)} a_{n} .
$$

Following the similar analysis above, also conferring to [5], it is a sufficient condition to guarantee normalizability of the eigenfunctions, i.e.,

$$
E_{n}=\omega_{L}(n+|m|+m)-\frac{1}{2} \frac{k^{2}}{\omega_{L}^{2}}
$$

and

$$
a_{n}=F\left(|m|, n, E, \omega_{L}, k, Z\right) a_{0}=0 .
$$

For $a_{0} \neq 0$, we can deduce

$$
F\left(|m|, n, E, \omega_{L}, k, Z\right)=0 .
$$

Substituting Eq.(53)into above Eq.(54), we obtain

$$
F\left(|m|, n, \omega_{L}(n+|m|+m)-\frac{1}{2} \frac{k^{2}}{\omega_{L}^{2}}, \omega_{L}, k, Z\right)=0 .
$$

From it, we obtain a constraint for $\omega_{L}$ and $k$, in another word, $\omega_{L}$ and $k$ can't be chosen arbitrarily. Furthermore, from Eq.(45), Eq.(46) and Eq.(52), we can get the polynomials of $\varphi$, i.e. Eq.(44). By use of Eq.(37) and Eq.(42), we can get the corresponding $R_{n}$.

In conclusion, we have solved the Eq.(4) by an analytical method. In the following section, we will build the connection between the radial part equation and the sextic oscillator with a centrifugal potential.

\section{KS TRANSFORMATION}

Via KS transformation, i.e.,

$$
\left\{\begin{array}{l}
r=\rho^{2} \\
\theta=2 \varphi
\end{array},\right.
$$

the Eq.(2) becomes

$$
\frac{1}{2}\left[-\frac{1}{4 \rho^{2}}\left(\frac{\partial^{2}}{\partial \rho^{2}}+\frac{1}{\rho} \frac{\partial}{\partial \rho}+\frac{1}{\rho^{2}} \frac{\partial^{2}}{\partial \varphi^{2}}\right) \psi-i \omega_{L} \frac{\partial}{\partial \varphi} \psi+\omega_{L}^{2} \rho^{4} \psi\right]-\frac{Z}{\rho^{2}} \psi+k \rho^{2} \psi=E \psi
$$

Substituting

$$
\psi(\rho, \varphi)=\frac{1}{\sqrt{2 \pi}} e^{i \tilde{m} \varphi} \chi(\rho)
$$


into the above equation, where $\tilde{m}=0, \pm 1, \pm 2, \ldots$, we get

$$
-\frac{1}{2}\left(\frac{d^{2}}{d \rho^{2}}+\frac{1}{\rho} \frac{d}{d \rho}-\frac{\tilde{m}^{2}}{\rho^{2}}\right) \chi+\left(2 \tilde{m} \omega_{L}-4 E\right) \rho^{2} \chi+4 k \rho^{4} \psi+2 \omega_{L}^{2} \rho^{6} \chi=4 Z \chi .
$$

In order to cancel the item which contains $\frac{1}{\rho}$, substituting

$$
\chi=\rho^{-\frac{1}{2}} \zeta
$$

into the above equation, one obtains

$$
-\frac{1}{2} \frac{d^{2} \zeta}{d \rho^{2}}+\frac{4 \tilde{m}^{2}-1}{8} \frac{1}{\rho^{2}} \zeta+\left(2 \omega_{L} \tilde{m}-4 E\right) \rho^{2} \zeta+4 k \rho^{4} \psi+2 \omega_{L}^{2} \rho^{6} \zeta=4 Z \zeta,
$$

which is the so-called sextic oscillator with a centrifugal barrier [6]. By comparison between Eq.(3) and Eq.(57), we can get

$$
m=\frac{\tilde{m}}{2}
$$

and

$$
\chi(\rho)=R(r) .
$$

Substituting Eq.(58) into the above Eq.(61), we obtain

$$
\zeta=\sqrt{\rho} R(r)
$$

which is precisely the wave function of the sextic oscillator with a centrifugal barrier. Moreover, using Eq.(56) and Eq.(60), we can replace the parameters in Eq.(62) by the counterparts used in Eq.(59). In section two and three, we have calculate $Z$ by two different methods. Therefore, it is very easy to get the eigenvalue $4 Z$ of Eq.(59).

\section{CONCLUSION AND ACKNOWLEDGMENTS}

In this paper, we have researched the two-dimensional hydrogen with a linear potential in a homogeneous magnetic field by two different methods, i.e., lie algebraic and analytic methods respectively. Furthermore we have also build the connection between the radial part of the schrödinger equation and the sextic oscillator with centrifugal barrier.

This work was supported in part by NSF of China(Grants No.10605013 and No.10975075).

[1] M. A. Shifman, Int. J. Mod. A 4 (1989) 2897. 
[2] A. V. Turbiner, Commun. Math. Phys. 118 (1988) 467.

[3] J. L. Chen, L. C. Kwek, C. H. Oh, and Y. Liu, J. Phys. A: Math. Gen. 34 (2001) 8889.

[4] M. A. Shifman, Int. J. Mod. A 4 (1989) 3311.

[5] M. Taut, J. Phys. A Math. Gen. 28 (1995) 2081.

[6] G. Levai, Phys. Rev. C 69 (2004) 014304. 